Research Paper

\title{
Identification of Candidate Olfactory Genes in Chilo suppressalis by Antennal Transcriptome Analysis
}

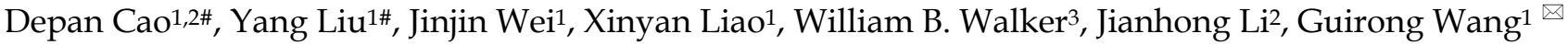 \\ 1. State Key Laboratory for Biology of Plant Diseases and Insect Pests, Institute of Plant Protection, Chinese Academy of Agricultural \\ Sciences, Beijing, China \\ 2. Laboratory of Pesticide, College of Plant Science \& Technology, Huazhong Agricultural University, Wuhan, China \\ 3. Swedish University of Agricultural Sciences, Department of Plant Protection Biology, Chemical Ecology Research Group, Alnarp, Swe- \\ den \\ \# These authors contributed equally to this work.
}

$\triangle$ Corresponding author: grwang@ippcaas.cn (G.R. Wang)

( ) Ivyspring International Publisher. This is an open-access article distributed under the terms of the Creative Commons License (http://creativecommons.org/ licenses/by-nc-nd/3.0/). Reproduction is permitted for personal, noncommercial use, provided that the article is in whole, unmodified, and properly cited.

Received: 2014.04.04; Accepted: 2014.05.25; Published: 20I4.07.26

\begin{abstract}
Antennal olfaction, which is extremely important for insect survival, mediates key behaviors such as host preference, mate choice, and oviposition site selection. In insects, odor detection is mediated by multiple proteins in the antenna, especially the odorant receptors (ORs) and ionotropic receptors (IRs), which ensure the specificity of the olfactory sensory neuron responses. In this study, we identified the olfactory gene repertoire of the rice stem borer, Chilo suppressalis, an economically important agricultural pest, which inflicts great damage to the rice yield in south and east part of Asia, especially in Southern China. By Illumina sequencing of male and female antennal transcriptomes, we identified 47 odorant receptors, 20 ionotropic receptors, 26 odorant binding proteins, $2 \mathrm{I}$ chemosensory proteins and 2 sensory neuron membrane proteins. Our findings make it possible for future research of the olfactory system of $C$. suppressalis at the molecular level.
\end{abstract}

Key words: C. suppressali, antennal olfaction, olfactory gene

\section{Introduction}

Chemical sensing is critically important to insect survival. For insects, olfaction, which is the primary sensory perception modality, is used to detect odor molecules in the environment. Olfaction guides the insect towards food, mating partners, and oviposition sites and also to facilitate detection of predators and toxic compounds [1]. The antenna is a specialized organ for insect sensing, especially for olfaction. Several types of sensilla, which are specialized hair-like, multi-pore structures, cover the surface of the antennae. Olfactory receptor neurons (ORNs) and auxiliary structures are housed within the antennae, positioned at the sensilla root [2]. For most of the olfactory sensilla, each hosts 1-4 ORNs, which extend their dendrites up into the sensilla and project their axons into the antennal lymph on towards the brain [3]. The
ORNs convert ecologically relevant volatile chemicals into an electrical impulse, which is transported to the primary olfactory center of the brain, the antennal lobe [4]. Within the sensilla-ORN structure, a number of gene families have been identified to play active roles in olfaction. These include the odorant binding proteins (OBPs), chemosensory proteins (CSPs), odorant receptors (ORs) ionotropic receptors (IRs), and the sensory neuron membrane proteins (SNMPs).

OBPs are hydrophilic soluble proteins that are secreted by the accessory cells around the ORNs and accumulate in the sensilla lymph [5]. OBPs are thought to be the first proteins that participate in the olfactory signal transduction procedure [6]. It is postulated that as the odor molecules diffuse through pores on sensilla, the soluble OBPs in sensillum 
lymph fluid selectively bind the liposoluble odor molecules [7] and transport them through the sensillum lymph to the surface of ORN dendrites [8,9]. OBPs are also thought to be directly involved in the activation of ORx/Orco complex in the recognition of some special odors $[10,11]$. Like OBPs, the CSPs are small soluble proteins that are enriched in the sensillum lymph, but also expressing broadly in non-olfactory tissues. The olfactory properties of CSPs are quite clear, for they bind odorant or pheromone compounds [12], but little is known about how CSPs function in olfactory system. Moreover, the broad tissues expression of CSPs implies unknown roles in non-olfactory procedures.

ORs are trans-membrane proteins located in the dendrite membrane of ORNs. Insect ORs are seven-transmembrane domain proteins [13] with a reversed membrane topology (intracellular $\mathrm{N}$-terminus) compared to the G-protein coupled vertebrate ORs [14]. In chemosensory signal transduction process, ORs play a central role as a bio-transducer, facilitating the conversion of the chemical message to an electrical signal. In this system, it is generally thought that in disparate individual OSNs, a single variable, ligand-binding ORx and a highly conserved, non-ligand binding Orco protein make up a stand-alone heteromeric structure that functions as a ligand-gated ion channel [14-17].

IRs make up a recently discovered ionotropic glutamate receptor (iGluR) -like protein family which has been shown to be involved in chemosensation [18]. The insect IRs contain structural regions that are conserved in iGluRs, namely, three transmembrane domains (M1, M2 and M3), a bipartite ligand-binding domain with two lobes (S1 and S2) and one ion channel pore $(\mathrm{P})$. But the conserved iGluR glutamate binding residues in $\mathrm{S} 1$ and $\mathrm{S} 2$ lobes are not retained in IRs, indicating their atypical binding characters [19]. Unlike the exclusive ORs, two or three IR genes were always co-expressed with one or both of the conserved IR8a and IR25a in one IR-expressing neuron [18]. Furthermore, IRs are thought to be a class of receptors far more ancient than OR families that animals use for sensing chemicals in the surrounding environment; this gene family has an extensive distribution, as it is found in mollusks, annelid and nematodes [20], and it displays a relatively high homology across species [21].

In this study, we sequenced and analyzed Chilo suppressalis adult antennal transcriptomes using Illumina sequencing. Our goals were to identify olfaction-related genes of this pest insect species, which is destructive to the rice farming in China, across Asia and in the Pacific. We report the results including sequencing, gene annotation, GO annotation and specifically, a set of 47 ORs, 20 IRs, 26 OBPs, 21 CSPs and 2 SNMPs.

\section{Results}

\section{Transcriptome overview}

With utilization of a 90PE RNA-Seq strategy by Illumina HiSeq 2000, about 56.4 million and 58.8 million raw-reads were obtained respectively from the libraries of male and female antenna. After filtering, 53.4 million and 55.3 million clean-reads comprised of 4.8 and 4.9 gigabases were generated for male and female antenna. Assemblies led to the generation of 79,706 and 77,404 unigenes separately for male and female. After merging and clustering, a final transcript dataset was revealed, with 66,560 unigenes consisting of 15,462 distinct clusters and 51,098 distinct singletons. The dataset was 50.63 megabases in size and with a mean length of 761nt and N50 of $1,271 \mathrm{nt}$. 11,849 unigenes were larger than 1,000nt in length, which comprised $17.80 \%$ of all unigenes (Table $1)$.

Through annotation by blastx, 30,232 (45.4\%) unigenes matched to known proteins; the remaining unigenes failed to match against any sequence with an e-value $<1$ e- 5 in neither of the nr nor SwissProt databases. Among the annotated unigenes, $70.4 \%$ had a best blast match to Lepidopteran sequences, primarily Danaus plexippus (59.2\%), and Bombyx mori (7.4\%) (Figure 1A). $52.0 \%$ of the annotated unigenes showed strong homology, with e-value $<1 \mathrm{e}-45$.

Table I. Assembly summary of $C$. suppressalis antenna transcriptome

\begin{tabular}{llllllll}
\hline & Sample & Total Number & Total Length(nt) & Mean Length(nt) & N50 & $\begin{array}{l}\text { Consensus } \\
\text { Sequences }\end{array}$ & $\begin{array}{l}\text { Distinct } \\
\text { Clusters }\end{array}$ \\
\hline Contig & Female & 130,229 & $44,138,907$ & 339 & 5 & - \\
& Male & 133,394 & $44,421,350$ & 333 & 569 & - \\
Singletons
\end{tabular}




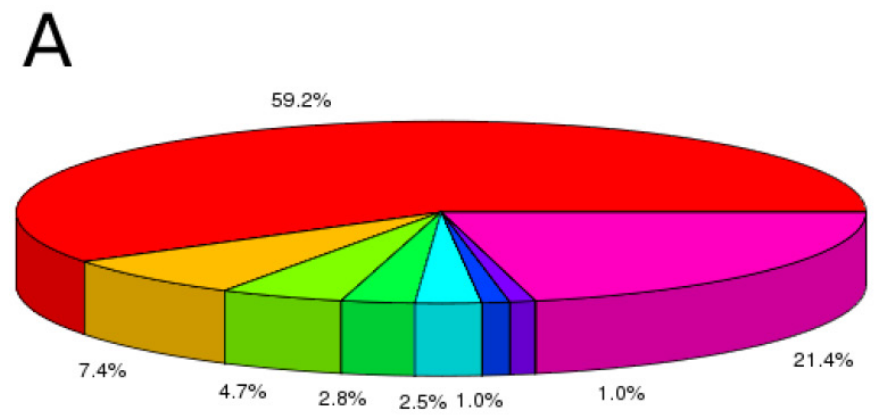

- Danaus plexippus

- Bombyx mori

- Tribolium castaneum

- Papilio xuthus

- Acyrthosiphon pisum

- Nasonia vitripennis

- Papilio polytes

n other

B

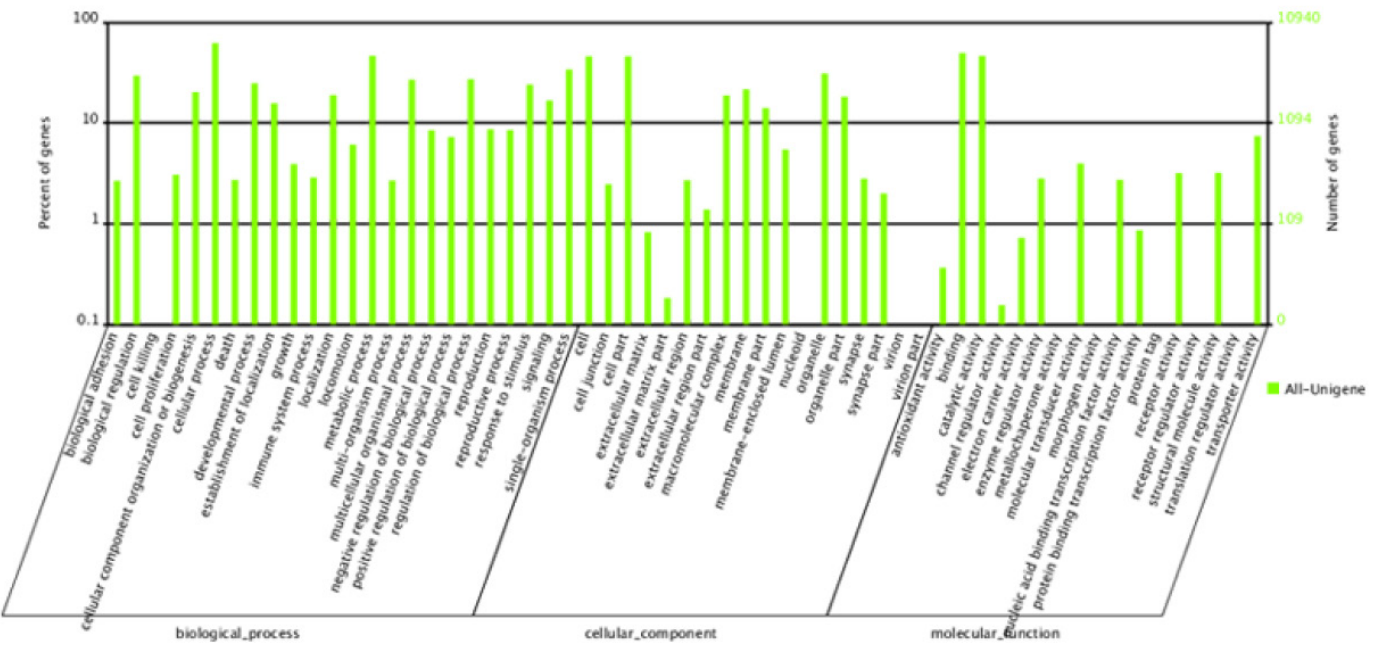

Figure I. Annotation summary of $C$. suppressalis antenna unigenes. (A) Species distribution of unigenes' best-hit annotation term in nr database. (B) Gene ontology classifications of the $C$. suppressalis unigenes.

Gene ontology (GO) annotation of the unigenes was obtained using Blast2GO pipeline according to the blastx search against nr. From the 66,560 final unigenes set, a total of 10,940 unigenes were assigned various GO terms. In the molecular function category, the genes expressed in the antennae were mostly enriched to molecular binding activity (e.g., nucleotide, ion and odorant binding) and catalytic activity (e.g., hydrolase and oxidoreductase). In the biological process terms, cellular and metabolic processes were the most represented. In the cellular component terms, cell, cell part and organelle were the most abundant (Figure 1B).

\section{Identification of Candidate Chemosensory Receptors}

The unigenes related to candidate chemosensory receptors were identified by keyword search of the blastx annotation. The predicted protein sequences of the unigenes were further searched by PSI-blastp with known Lepidopteran chemosensory receptors [4] to indentify more candidate ORs. We identified 47 distinct unigenes that were putative OR genes. Of these,
23 sequences were full-length OR genes because they have intact open reading frames with a general length of 1,200bp and 5-7 transmembrane domains, which are characteristic of typical insect ORs.

The C. suppressalis Orco co-receptor orthologue was easily detected as it has a high degree of identity with the conserved insect co-receptor: this gene was named CsupOrco. Six unigenes were considered to be putative pheromone receptors because they shared considerable similarity with previously characterized Lepidopteran pheromone receptors and were clustered together into one subgroup in the phylogenetic tree (Figure 2). These 6 candidate ORs were named as "CsupPRx" ( $x=1$ through 6), to more clearly indicate function, as previously reported Lepidopteran pheromone receptors were not clearly separated from the general odorant receptors and followed no orderly numbering system. The naming convention followed in this report is also consistent with general naming of odorant binding proteins, where the pheromone binding proteins (PBPs) are distinguished from other odorant binding proteins (OBPs). Other candidate ORs were highly divergent and shared low similarity 
with other insect ORs, which is common for insect olfactory receptor genes. These genes were named as "CsupOR", followed by a numeral, in descending order of their coding region lengths.

Phylogenetic analysis was performed with ORs from $B$. mori, $H$. armigera, $H$. virescens, and PR sequences from P. xylostella and some Crambidae insects. For the relatively conserved PR genes, the CsupPR4 and CsupPR5 were clustered together with the Crambidae pheromone receptor 1 and 3. CsupPR1, 2, 3 and 6 were not closely grouped with the Crambidae PRs but clustered with the $P$. xylostella PR clade with high bootstrap support. Almost all CsupOR candidates clustered with at least one Lepidopteran orthologous gene in the phylogenetic tree. No C. suppressalis specific OR family expansion was discovered in our phylogenetic tree.
Information including unigene reference, length, and best blast $x$ hit of all 47 odorant receptors are listed in Table 2. The sequences are listed in Additional File 1: Supplementary Material S1.

\section{Identification of Candidate Ionotropic Receptors}

The putative IR genes in the C. suppressalis antennal transcriptome were represented according to their similarity to known insect IRs. Bioinformatic analysis led to the identification of 20 candidates IRs, in which 13 sequences contain a full-length ORF, the remaining 7 sequences are marked as incomplete due to lacking a complete $5^{\prime}$ or $3^{\prime}$ terminus. The insect IRs contained three transmembrane domains. TMHMM2.0 predicted 10 IR candidates with three transmembrane domains (Table 3 ).

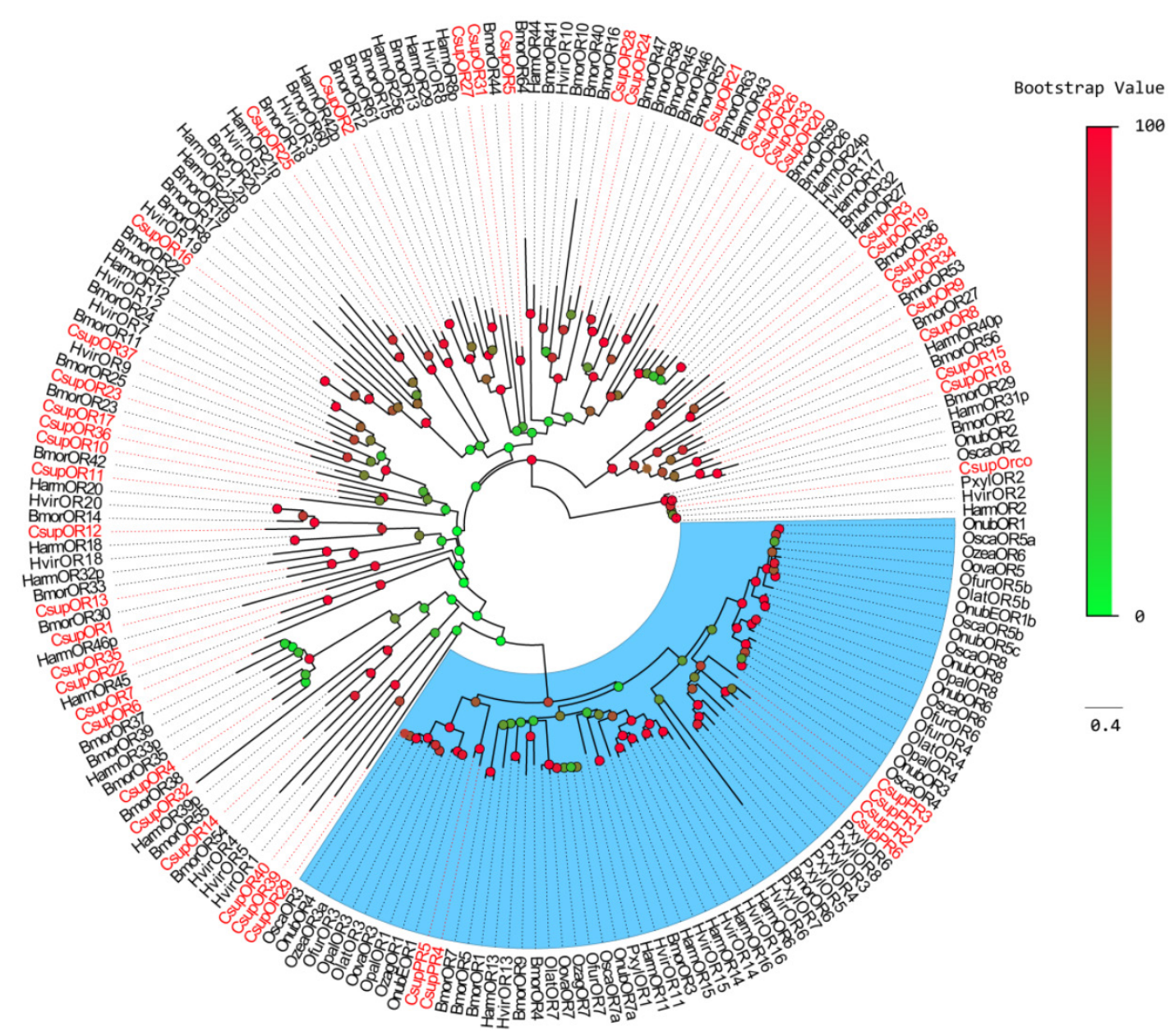

Figure 2. Phylogenetic tree of candidate CsupORs with known lepidopteran OR sequences. Harm: $H$. armigera; Hvir: $H$. virescens; Bmor: $B$. mori; Pxyl: P. xylostella; Osca: Ostrinia scapulalis; Onub: Ostrinia nubilalis; Ozea: Ostrinia zealis; Ofur: Ostrinia furnacalis; Opal: Ostrinia palustralis; Ozag: Ostrinia zaguliaevi: Oova: Ostrinia ovalipennis; Olat: Ostrinia latipennis. The clade in blue indicates the pheromone receptor gene clade. 
Table 2. Unigenes of candidate olfactory receptors

\begin{tabular}{|c|c|c|c|c|c|c|c|}
\hline Gene name & $\begin{array}{l}\text { Length } \\
\text { (nt) }\end{array}$ & ORF (aa) & $\begin{array}{l}\text { Unigene refer- } \\
\text { ence }\end{array}$ & Status & $\begin{array}{l}\text { TMD } \\
\text { (No.) }\end{array}$ & Evalue & BLASTx best hit \\
\hline \multicolumn{8}{|l|}{$\overline{\text { ORco }}$} \\
\hline CsupOrco & 3864 & 474 & CL5509.Contig1 & Complete ORF & 7 & $0.0 \mathrm{E}+00$ & $\mathrm{gb}$ | AFQ94048.1 | olfactory receptor 2 [Chilo suppressalis] \\
\hline \multicolumn{8}{|c|}{ Pheromone receptor } \\
\hline CsupPR1 & 1492 & 424 & Unigene14957 & Complete ORF & 6 & $1.0 \mathrm{E}-94$ & $\mathrm{gb}$ |ADB89183.1 | odorant receptor 6 [Ostrinia nubilalis] \\
\hline CsupPR2 & 2220 & 367 & Unigene18611 & Complete ORF & 4 & $2.0 \mathrm{E}-95$ & gb | ADB89183.1 | odorant receptor 6 [Ostrinia nubilalis] \\
\hline CsupPR3 & 2044 & 264 & CL1103.Contig5 & Complete ORF & 6 & $1.0 \mathrm{E}-56$ & $\mathrm{gb}$ | AFK30402.1 | E-race odorant receptor 6 [Ostrinia nubilalis] \\
\hline CsupPR4 & 483 & 160 & CL812.Contig2 & $5^{\prime}, 3^{\prime}$ lost & 2 & $2.0 \mathrm{E}-28$ & dbj| BAG71417.1 | olfactory receptor-1 [Diaphania indica] \\
\hline CsupPR5 & 297 & 99 & CL4759.Contig1 & $5^{\prime}, 3^{\prime}$ lost & 1 & $2.0 \mathrm{E}-21$ & dbj| BAH57981.1 | olfactory receptor [Ostrinia latipennis] \\
\hline CsupPR6 & 259 & 86 & Unigene43713 & $5^{\prime}, 3^{\prime}$ lost & 2 & $6.0 \mathrm{E}-10$ & $\mathrm{gb}$ | AFK30403.1 | odorant receptor 6 [Ostrinia furnacalis] \\
\hline \multicolumn{8}{|c|}{ Other odorant receptor } \\
\hline CsupOR1 & 1537 & 457 & Unigene28449 & Complete ORF & 7 & $5.0 \mathrm{E}-152$ & ref |NP_001116817.1 | olfactory receptor-like [Bombyx mori] \\
\hline CsupOR2 & 1371 & 446 & Unigene15165 & Complete ORF & 6 & 2.0E-179 & ref |NP_001155301.1 | olfactory receptor 60 [Bombyx mori] \\
\hline CsupOR3 & 1612 & 439 & Unigene29790 & Complete ORF & 5 & $5.0 \mathrm{E}-56$ & dbj|BAH66328.1 | olfactory receptor [Bombyx mori] \\
\hline CsupOR4 & 1443 & 432 & CL3655.Contig2 & Complete ORF & 6 & 1.0E-140 & $\mathrm{gb}$ |AFL70813.1 | odorant receptor 50, partial [Manduca sexta] \\
\hline CsupOR5 & 1445 & 429 & Unigene22904 & Complete ORF & 7 & $4.0 \mathrm{E}-141$ & ref | NP_001166607.1 | olfactory receptor 44 [Bombyx mori] \\
\hline CsupOR6 & 1381 & 423 & CL5260.Contig2 & Complete ORF & 6 & $1.0 \mathrm{E}-131$ & $\mathrm{gb}$ | AFL70813.1 | odorant receptor 50, partial [Manduca sexta] \\
\hline CsupOR7 & 2645 & 423 & Unigene26044 & Complete ORF & 4 & $2.0 \mathrm{E}-27$ & 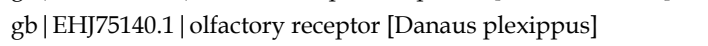 \\
\hline CsupOR8 & 1423 & 422 & Unigene990 & Complete ORF & 5 & 3.0E-114 & ref | NP_001166893.1 | olfactory receptor 27 [Bombyx mori] \\
\hline CsupOR9 & 1429 & 422 & CL3918.Contig4 & $5^{\prime}$ lost & 6 & 4.0E-112 & $\begin{array}{l}\text { gb | AFC91736.1 | putative odorant receptor OR28 [Cydia pomo- } \\
\text { nella] }\end{array}$ \\
\hline CsupOR10 & 1600 & 414 & Unigene24741 & $5^{\prime}$ lost & 7 & $4.0 \mathrm{E}-116$ & ref | NP_001091818.1 | olfactory receptor 42 [Bombyx mori] \\
\hline CsupOR11 & 1270 & 406 & CL5145.Contig2 & Complete ORF & 6 & $1.0 \mathrm{E}-10$ & sp | P81922 | Odorant receptor 47b [Drosophila melanogaster] \\
\hline CsupOR12 & 1501 & 402 & Unigene11744 & Complete ORF & 5 & 4.0E-116 & $\begin{array}{l}\text { gb | ACC63240.1 | olfactory receptor 20, partial [Helicoverpa } \\
\text { armigera] }\end{array}$ \\
\hline CsupOR13 & 1318 & 402 & CL2287.Contig1 & Complete ORF & 7 & 7.0E-109 & $\begin{array}{l}\text { tpg |DAA05986.1 | TPA: TPA_exp: odorant receptor } 30 \text { [Bombyx } \\
\text { mori] }\end{array}$ \\
\hline CsupOR14 & 1209 & 400 & Unigene4576 & $5^{\prime}$ lost & 6 & $1.0 \mathrm{E}-119$ & ref |NP_001166616.1 | olfactory receptor 54 [Bombyx mori] \\
\hline CsupOR15 & 1275 & 400 & Unigene35932 & Complete ORF & 5 & $1.0 \mathrm{E}-150$ & ref |NP_001166617.1 | olfactory receptor 56 [Bombyx mori] \\
\hline CsupOR16 & 1344 & 397 & Unigene33520 & $3^{\prime}$ lost & 6 & $3.0 \mathrm{E}-135$ & ref |NP_001166613.1 | olfactory receptor 22 [Bombyx mori] \\
\hline CsupOR17 & 1365 & 397 & CL458.Contig1 & Complete ORF & 6 & $6.0 \mathrm{E}-162$ & $\begin{array}{l}\text { gb |AFC91721.1 | putative odorant receptor OR12 [Cydia pomo- } \\
\text { nella] }\end{array}$ \\
\hline CsupOR18 & 1442 & 397 & CL3235.Contig2 & $3^{\prime}$ lost & 6 & $7.0 \mathrm{E}-134$ & ref | NP_001166894.1 | olfactory receptor 29 [Bombyx mori] \\
\hline CsupOR19 & 1632 & 395 & CL545.Contig1 & Complete ORF & 5 & $8.0 \mathrm{E}-54$ & gb | EHJ63141.1 | olfactory receptor [Danaus plexippus] \\
\hline CsupOR20 & 1382 & 390 & CL1707.Contig3 & Complete ORF & 5 & $6.0 \mathrm{E}-124$ & $\begin{array}{l}\text { emb |CAG38118.1 | putative chemosensory receptor } 17 \text { [Heliothis } \\
\text { virescens] }\end{array}$ \\
\hline CsupOR21 & 1220 & 386 & CL727.Contig1 & Complete ORF & 5 & 2.0E-94 & ref | NP_001166620.1 | olfactory receptor 63 [Bombyx mori] \\
\hline CsupOR22 & 1388 & 381 & Unigene18694 & Complete ORF & 6 & 4.0E-118 & $\begin{array}{l}\text { gb|AFC91732.1 | putative odorant receptor OR24 [Cydia pomo- } \\
\text { nella] }\end{array}$ \\
\hline CsupOR23 & 1193 & 379 & Unigene18626 & $5^{\prime}$ lost & 6 & $9.0 \mathrm{E}-60$ & ref | NP_001166606.1 | olfactory receptor 23 [Bombyx mori] \\
\hline CsupOR24 & 1270 & 378 & CL380.Contig1 & Complete ORF & 4 & $3.0 \mathrm{E}-78$ & $\begin{array}{l}\text { gb|AFC91719.1 | putative odorant receptor OR10 [Cydia pomo- } \\
\text { nella] }\end{array}$ \\
\hline CsupOR25 & 863 & 377 & Unigene17554 & $5^{\prime}$ lost & 2 & $3.0 \mathrm{E}-86$ & ref | NP_001166895.1 | olfactory receptor 18 [Bombyx mori] \\
\hline CsupOR26 & 1214 & 375 & Unigene22379 & $5^{\prime}$ lost & 4 & $2.0 \mathrm{E}-54$ & $\begin{array}{l}\text { ref |NP_001091790.1 | candidate olfactory receptor [Bombyx } \\
\text { mori] }\end{array}$ \\
\hline CsupOR27 & 545 & 370 & Unigene24576 & $5^{\prime}$ lost & 4 & $1.0 \mathrm{E}-26$ & $\begin{array}{l}\text { tpg |DAA05974.1 | TPA: TPA_exp: odorant receptor } 15 \text { [Bombyx } \\
\text { mori] }\end{array}$ \\
\hline CsupOR28 & 1061 & 353 & Unigene22927 & $5^{\prime}, 3^{\prime}$ lost & 6 & $3.0 \mathrm{E}-135$ & ref |NP_001104832.2 | olfactory receptor 16 [Bombyx mori] \\
\hline CsupOR29 & 1060 & 348 & Unigene33676 & $5^{\prime}$ lost & 6 & $4.0 \mathrm{E}-118$ & $\mathrm{gb}$ | AEF32141.1 | odorant receptor [Spodoptera exigua] \\
\hline CsupOR30 & 1170 & 346 & CL1602.Contig3 & $5^{\prime}$ lost & 5 & $2.0 \mathrm{E}-67$ & $\begin{array}{l}\text { gb | AFC91739.1 | putative odorant receptor OR31 [Cydia pomo- } \\
\text { nella] }\end{array}$ \\
\hline CsupOR31 & 1022 & 340 & Unigene28661 & $5^{\prime}, 3^{\prime}$ lost & 6 & $1.0 \mathrm{E}-26$ & $\mathrm{gb}$ | EHJ65088.1 | olfactory receptor 44 [Danaus plexippus] \\
\hline CsupOR32 & 2686 & 332 & CL4999.Contig1 & Complete ORF & 6 & $3.0 \mathrm{E}-43$ & $\begin{array}{l}\text { ref |NP_001091791.1 | candidate olfactory receptor [Bombyx } \\
\text { mori] }\end{array}$ \\
\hline CsupOR33 & 971 & 323 & Unigene 30218 & $5^{\prime}, 3^{\prime}$ lost & 4 & $8.0 \mathrm{E}-87$ & $\begin{array}{l}\text { ref |NP_001091790.1 | candidate olfactory receptor [Bombyx } \\
\text { mori] }\end{array}$ \\
\hline CsupOR34 & 1000 & 295 & Unigene35881 & $5^{\prime}$ lost & 4 & $8.0 \mathrm{E}-82$ & ref | NP_001166892.1 | olfactory receptor 36 [Bombyx mori] \\
\hline CsupOR35 & 911 & 272 & CL5748.Contig2 & $5^{\prime}$ lost & 2 & $6.0 \mathrm{E}-76$ & $\begin{array}{l}\text { gb | AFC91725.1 | putative odorant receptor OR17 [Cydia pomo- } \\
\text { nella] }\end{array}$ \\
\hline CsupOR36 & 793 & 264 & Unigene26834 & $5^{\prime}, 3^{\prime}$ lost & 4 & $5.0 \mathrm{E}-65$ & ref | NP_001091818.1 | olfactory receptor 42 [Bombyx mori] \\
\hline CsupOR37 & 834 & 245 & CL296.Contig2 & $5^{\prime}$ lost & 2 & $1.0 \mathrm{E}-66$ & dbj| BAH66322.1 | olfactory receptor [Bombyx mori] \\
\hline CsupOR38 & 657 & 219 & Unigene35370 & $5^{\prime}, 3^{\prime}$ lost & 4 & $1.0 \mathrm{E}-72$ & ref | NP_001166892.1 | olfactory receptor 36 [Bombyx mori] \\
\hline CsupOR39 & 604 & 163 & CL4235.Contig2 & $3^{\prime}$ lost & 3 & $3.0 \mathrm{E}-16$ & dbj| BAH66322.1 | olfactory receptor [Bombyx mori] \\
\hline CsupOR40 & 406 & 120 & CL4235.Contig1 & $3^{\prime}$ lost & 2 & $1.0 \mathrm{E}-11$ & ref |NP_001104828.1 | olfactory receptor 25 [Bombyx mori] \\
\hline
\end{tabular}


Table 3. Unigenes of candidate ionotropic receptors

\begin{tabular}{|c|c|c|c|c|c|c|c|}
\hline Gene name & $\begin{array}{l}\text { Lengt } \\
\mathrm{h}(\mathrm{nt})\end{array}$ & $\begin{array}{l}\text { ORF } \\
\text { (aa) }\end{array}$ & $\begin{array}{l}\text { Unigene } \\
\text { reference }\end{array}$ & Status & $\begin{array}{l}\text { TMD } \\
\text { (No.) }\end{array}$ & Evalue & BLAST $x$ best hit \\
\hline CsupIR1 & 1934 & 574 & Unigene35421 & $5^{\prime}$ lost & 3 & $0.0 \mathrm{E}+00$ & $\begin{array}{l}\text { gb | EHJ72198.1 | putative ionotropic glutamate receptor-invertebrate [Danaus } \\
\text { plexippus] }\end{array}$ \\
\hline CsupIR1.1 & 2121 & 656 & CL4511.Contig3 & $\begin{array}{l}\text { Complete } \\
\text { ORF }\end{array}$ & 3 & $1.0 \mathrm{E}-113$ & $\begin{array}{l}\text { gb | EHJ72198.1 | putative ionotropic glutamate receptor-invertebrate [Danaus } \\
\text { plexippus] }\end{array}$ \\
\hline CsupIR2 & 2147 & 320 & CL2718.Contig3 & $\begin{array}{l}\text { Complete } \\
\text { ORF }\end{array}$ & 4 & $4.0 \mathrm{E}-163$ & gb |AAB62572.1 |GABA-gated chloride channel isoform a3 [Heliothis virescens] \\
\hline CsupIR21a & 2972 & 844 & Unigene11518 & $\begin{array}{l}\text { Complete } \\
\text { ORF }\end{array}$ & 3 & $0.0 \mathrm{E}+00$ & $\begin{array}{l}\text { gb | ADR64678.1 | putative chemosensory ionotropic receptor IR21a [Spodoptera } \\
\text { littoralis] }\end{array}$ \\
\hline CsupIR25a & 3304 & 927 & Unigene17452 & $\begin{array}{l}\text { Complete } \\
\text { ORF }\end{array}$ & 3 & $2.0 \mathrm{E}-75$ & sp | P39087 | Glutamate receptor, ionotropic kainate 2 [Mus musculus] \\
\hline CsupIR3 & 2100 & 474 & Unigene29712 & $\begin{array}{l}\text { Complete } \\
\text { ORF }\end{array}$ & 4 & $0.0 \mathrm{E}+00$ & gb | EHJ68597.1 | putative glycine receptor beta precursor [Danaus plexippus] \\
\hline CsupIR4 & 931 & 277 & Unigene904 & $5^{\prime}, 3^{\prime}$ lost & 2 & $5.0 \mathrm{E}-12$ & sp |Q68Y21 | Glutamate receptor delta-2 subunit [Danio rerio] \\
\hline CsupIR40a & 2918 & 707 & CL4571.Contig2 & $\begin{array}{l}\text { Complete } \\
\text { ORF }\end{array}$ & 3 & $1.0 \mathrm{E}-15$ & sp |Q9ULK0 | Glutamate receptor delta-1 subunit [Homo sapiens] \\
\hline CsupIR41a & 1989 & 598 & CL876.Contig2 & $5^{\prime}$ lost & 3 & $0.0 \mathrm{E}+00$ & gb | AFC91758.1 | putative ionotropic receptor IR41a [Cydia pomonella] \\
\hline CsupIR64a & 1258 & 380 & Unigene22885 & $5^{\prime}$ lost & 4 & $8.0 \mathrm{E}-17$ & sp |Q68Y21 | Glutamate receptor delta-2 subunit [Danio rerio] \\
\hline CsupIR68a & 2044 & 674 & Unigene14878 & $\begin{array}{l}\text { Complete } \\
\text { ORF }\end{array}$ & 6 & $0.0 \mathrm{E}+00$ & $\begin{array}{l}\text { gb | ADR64682.1 | putative chemosensory ionotropic receptor IR68a [Spodoptera } \\
\text { littoralis] }\end{array}$ \\
\hline CsupIR75d & 1145 & 294 & CL349.Contig2 & $5^{\prime}$ lost & 1 & $1.0 \mathrm{E}-11$ & sp | P34299 | Glutamate receptor 1 [Caenorhabditis elegans] \\
\hline CsupIR75p & 2200 & 615 & CL46.Contig4 & $\begin{array}{l}\text { Complete } \\
\text { ORF }\end{array}$ & 3 & $0.0 \mathrm{E}+00$ & $\begin{array}{l}\mathrm{gb} \mid \text { ADR64684.1 | putative chemosensory ionotropic receptor IR75p [Spodoptera } \\
\text { littoralis] }\end{array}$ \\
\hline CsupIR75p.1 & 1429 & 441 & CL2655.Contig2 & $5^{\prime}$ lost & 4 & $0.0 \mathrm{E}+00$ & $\begin{array}{l}\text { gb | EHJ72019.1 | putative ionotropic glutamate receptor-invertebrate [Danaus } \\
\text { plexippus] }\end{array}$ \\
\hline CsupIR75q1 & 588 & 196 & Unigene9838 & $5^{\prime}, 3^{\prime}$ lost & 2 & $1.0 \mathrm{E}-56$ & gb | AFC91752.1 | putative ionotropic receptor IR75q2 [Cydia pomonella] \\
\hline CsupIR75q2 & 2011 & 635 & CL1806.Contig1 & $\begin{array}{l}\text { Complete } \\
\text { ORF }\end{array}$ & 4 & $0.0 \mathrm{E}+00$ & gb | AFC91752.1 | putative ionotropic receptor IR75q2 [Cydia pomonella] \\
\hline CsupIR76b & 2070 & 547 & Unigene33212 & $\begin{array}{l}\text { Complete } \\
\text { ORF }\end{array}$ & 3 & $0.0 \mathrm{E}+00$ & gb | AFC91765.1 | putative ionotropic receptor IR76b [Cydia pomonella] \\
\hline CsupIR87a & 2067 & 647 & Unigene8213 & $\begin{array}{l}\text { Complete } \\
\text { ORF }\end{array}$ & 6 & 4.0E-07 & sp | O43424 | Glutamate receptor delta-2 subunit [Homo sapiens] \\
\hline CsupIR8a & 3058 & 912 & Unigene17458 & $\begin{array}{l}\text { Complete } \\
\text { ORF }\end{array}$ & 3 & $0.0 \mathrm{E}+00$ & gb | AFC91764.1 | putative ionotropic receptor IR8a, partial [Cydia pomonella] \\
\hline CsupIR93a & 2878 & 877 & CL2805.Contig1 & $\begin{array}{l}\text { Complete } \\
\text { ORF }\end{array}$ & 3 & $1.0 \mathrm{E}-33$ & sp |Q63226 | Glutamate receptor delta-2 subunit [Rattus norvegicus] \\
\hline
\end{tabular}

To distinguish putative IRs from iGluRs, putative C. suppressalis IRs were aligned with IR orthologoues from D. melanogaster, B. mori, S. littoralis and some D. melanogaster iGluRs for a phylogenetic analysis. The results revealed a clear segregation between DmeliGluRs and insect IRs (Figure 3). In the phylogenetic tree of IRs, most $C$. suppressalis IR candidates clustered with their ionotropic receptor orthologues into a separate clade. According to their positions in phylogenetic tree and strong bootstrap support, 15 of 20 candidate $C$. suppressalis IRs were given names consistent with the number and suffix of the Dmel/Bmor/Slit IR orthologues in the same clade.

Two of the remaining 5 IR sequences, CL4511.Contig3 and CL2655.Contig2, were clustered into the SlitIR1 and Slit/Bmor IR75p clades, respectively, with reliable bootstrap support, forming small expansions with the CsupIR1 and CsupIR75p genes. Considering that these two sequences contain typical IR characteristics, these two sequences may likely be C. suppressalis specific genes, or their orthologues haven't been detected in other insects. These two sequences were named as "CsupIR1.1" and "Csu-
pIR75p.1", respectively. The other 3 sequences, CL2718.Contig3, Unigene29712 and Unigene904, had low bootstrap values unable to clearly demonstrate their phylogenetic positions, were named as "CsupIR2", "CsupIR3" and "CsupIR4", respectively. The information including unigene reference, length, and best blast $x$ hit of all the 20 IRs are listed in Table 3. The sequences of all 20 IRs were listed in SAdditional File 1: upplementary Material S1.

\section{Identification of Putative Odorant-binding Proteins and Chemosensory Proteins}

In addition to keyword searching and PSI-Blast, we also used motif scanning to detect the conserved 6 cysteine residues pattern $\left(\mathrm{C} 1-\mathrm{X}_{5-39}-\mathrm{C} 2-\mathrm{X}_{3}-\mathrm{C} 3-\mathrm{X}_{21-44}-\mathrm{C} 4-\right.$ $\left.\mathrm{X}_{7-12-} \mathrm{C} 5-\mathrm{X}_{8}-\mathrm{C} 6\right)$ [22] of the putative odorant-binding proteins. In our transcript set, we identified 26 different sequences encoding odorant binding proteins, including 4 PBPs and 2 GOBPs. In these 26 sequences, 23 had intact ORFs detected; 3 unigenes failed in the signal peptide test which is performed by SignalP. Sequence alignment showed that almost all the putative OBPs shared the classic six-cysteine motif, except 
4 sequences (CsupOBP4, 10, 11 and 13), which grouped into the "minus- $C$ " subgroup with their second cysteine residues missing [23]. It was also notable that all 4 "minus-C" OBPs had a lysine residue in place of the $\mathrm{C} 2$ cysteine (Figure 4 ). In the phylogenetic tree, the PBP and GOBP sequences were clustered respectively into the PBP and GOBP clades as expected (Figure 5). All candidate OBP sequences were clustered with at least one lepidopteran orthologue. Comparing our putative OBPs with NCBI records of C. suppressalis, we identified 10 as "discovered genes", which are GOBP1, 2, PBP1, 2, 3, OBP2, 8, 13, 14 and 17. All of these "discovered genes" have identities over $96 \%$ in amino acid to their most similar NCBI records. Therefore, we named these candidate GOBPs and PBPs following the existing NCBI records. We named the candidate OBPs as "CsupOBP" followed by a numeral in descending order of their coding region lengths, as the numbering of existing $C$. suppressalis OBP records is confusing (Table 4).

Bioinformatic analysis led to the identification of 21 different sequences encoding candidate CSPs. Among them, 18 sequences have full-length ORFs and signal peptides; Due to incomplete N-termini, the remaining 3 failed in the SignalP test. The conserved cysteine pattern of $\mathrm{C} 1-\mathrm{X}_{6-8}-\mathrm{C} 2-\mathrm{X}_{18}-\mathrm{C} 3-\mathrm{X}_{2}-\mathrm{C} 4$ [24] and the six-helix secondary-structure were retained in all 21 candidate CSPs (Figure 6). Neighbor-joining tree analysis showed that all of the 21 sequences clustered with Lepidopteran orthologous genes (Figure 7). These candidate CSPs were named as "CsupCSP" followed by a numeral in descending order of their coding region lengths. The information on the CSPs is listed in Table 5. The sequences are listed in Additional File 1: Supplement Material S1.

\section{Identification of Candidate Sensory Neuron Membrane Proteins}

SNMPs are thought to be involved in the recognition of Lepidopteran pheromone, since they were first identified in Lepidopteran pheromone-sensitive neurons [25, 26]. SNMPs of two families, SNMP1 and 2 , were discovered in our C. suppressalis antennal transcriptome. Unigene35775 showed a 99\% identity to the CsupSNMP1 published in Genebank. And the CL173.contig15 covered the whole sequence of the CsupSNMP2 (GI: 406668637). Our SNMP unigene sequences are available in Additional File 1: Supplementary Material S1.

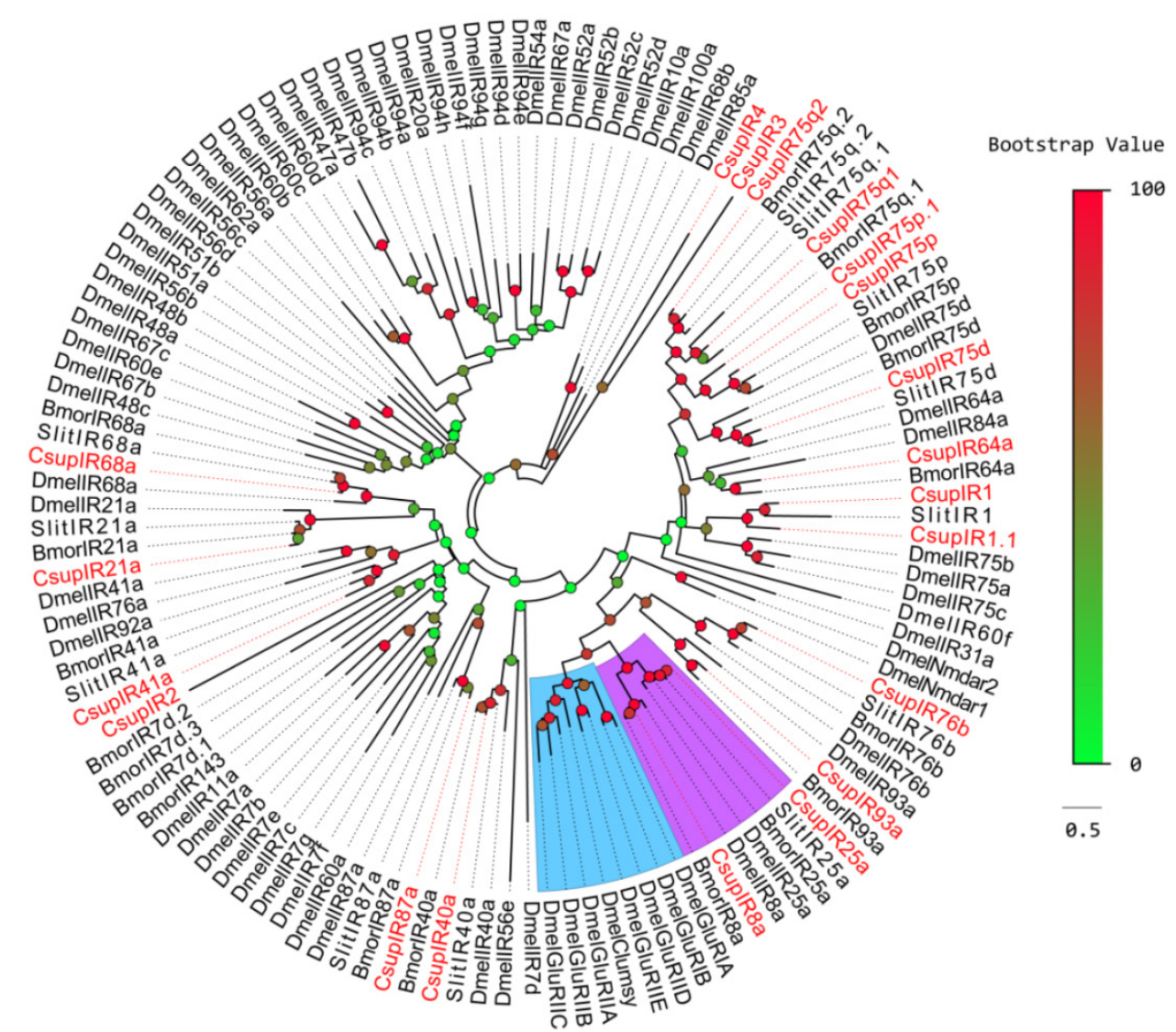

Figure 3. Phylogenetic tree of candidate CsupIRs with known lepidopteran IRs and iGluRs. Dmel: D. melanogaster,Bmor: B. mori, Slit: S. littoralis. The clade in blue indicates the iGluR gene clade; the clade in purple indicates the IR8a/IR25a clade. 

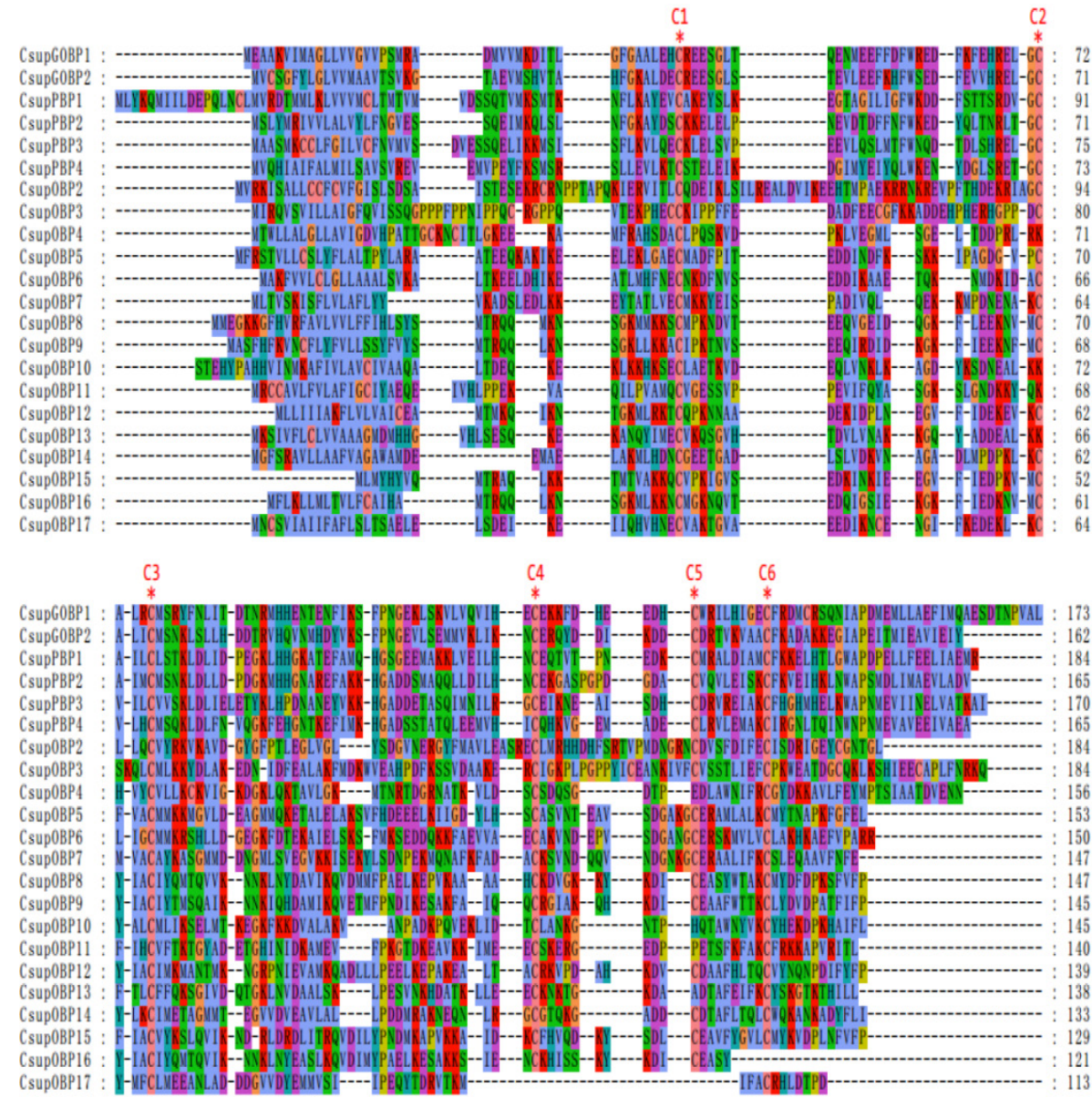

Figure 4. Sequences alignment of putative CsupOBPs. The conserved cysteine residues were marked with “*”. Because of the overly long sequence of CsupOBPI, the CsupOBPI is not included in the multisequence alignment.

Figure 5. Phylogenetic tree of candidate CsupOBPs with known lepidopteran OBPs. Bmor: B. mori, Hv: $H$. virescens. The clade in blue indicates the PBP gene clade; the clade in purple indicates the GOBP clade.

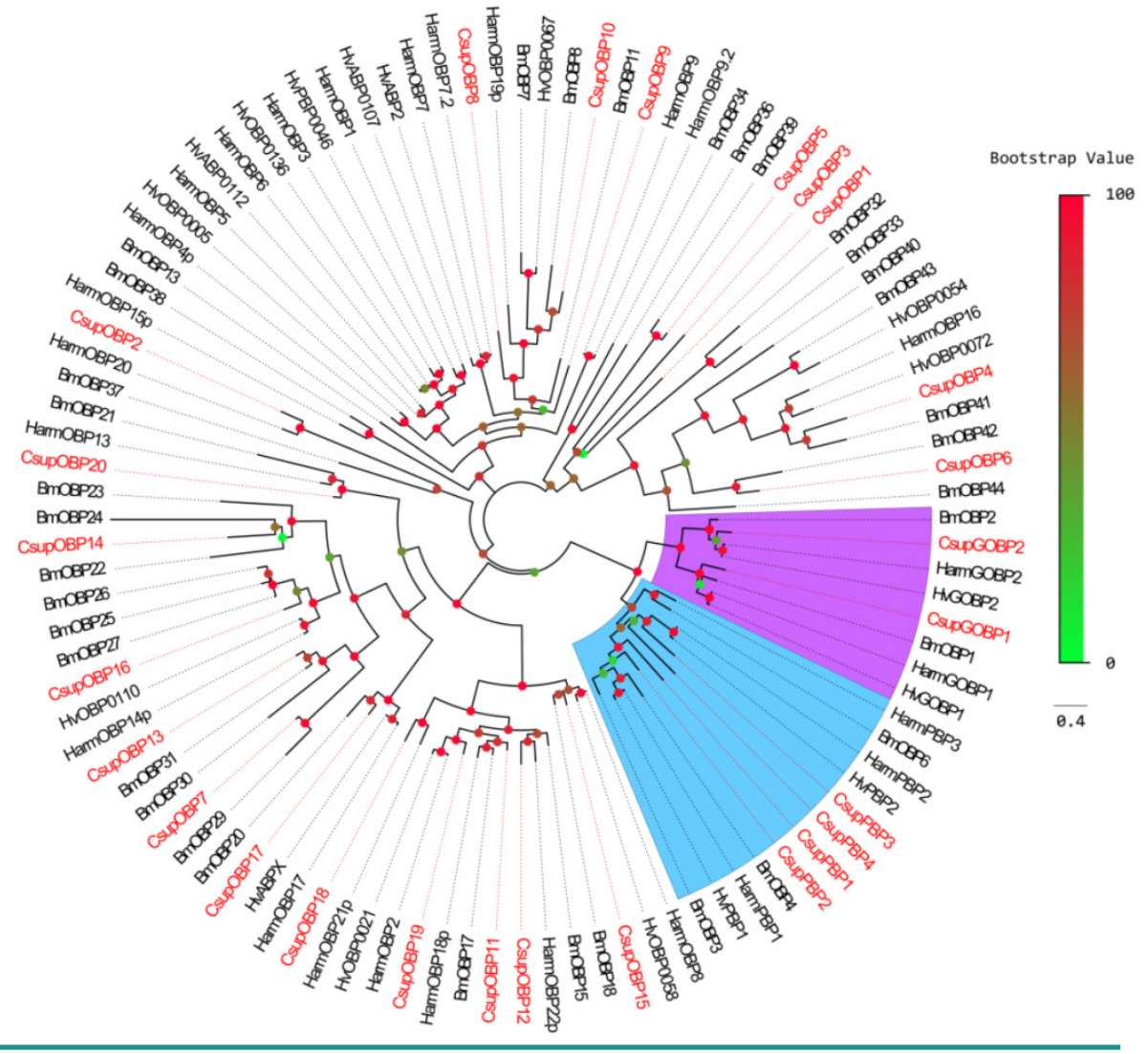

http://www.ijbs.com 

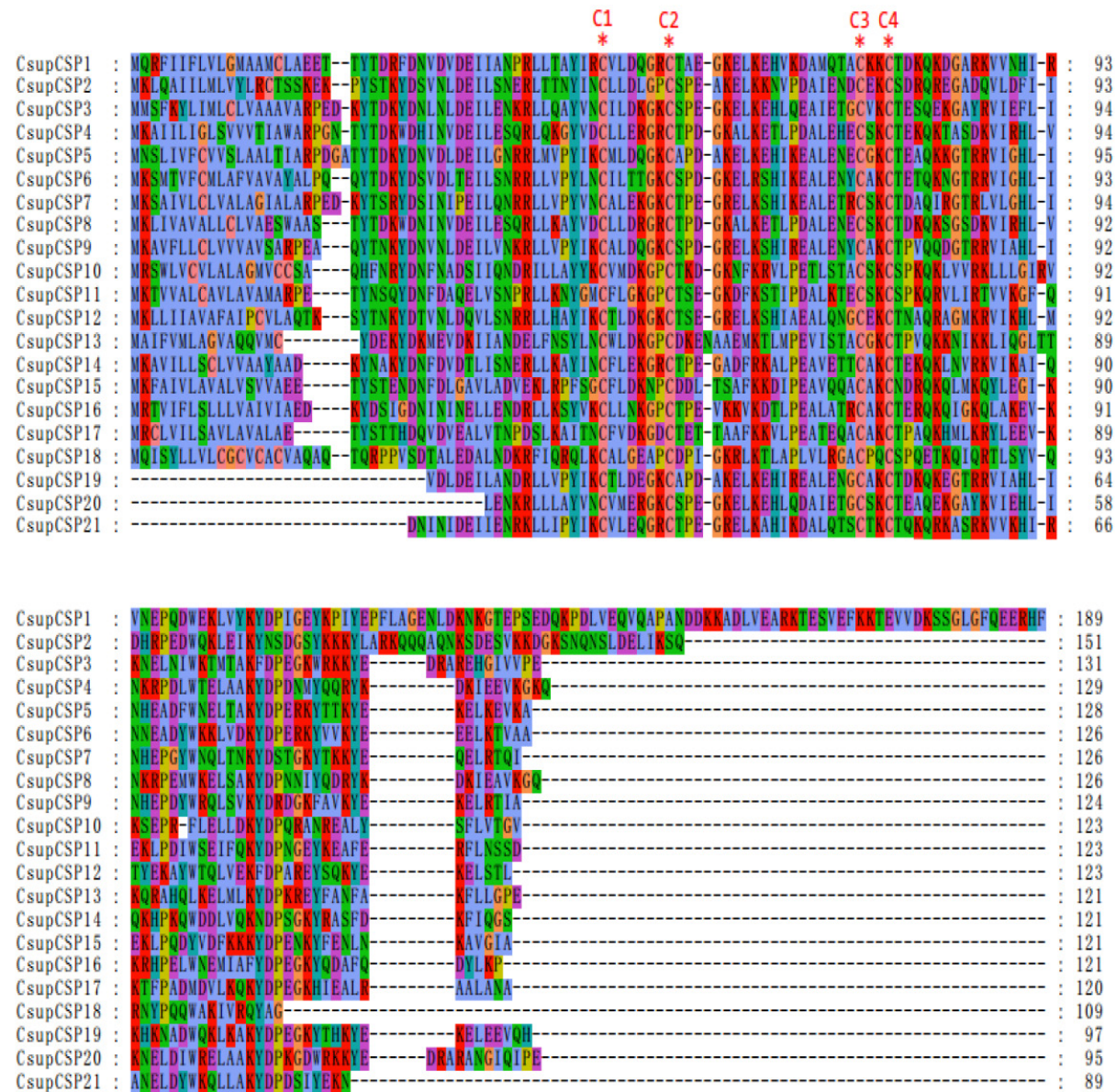

Figure 6 Sequences alignment of putative CsupCSPs. The conserved cysteine residues were marked with “*”.

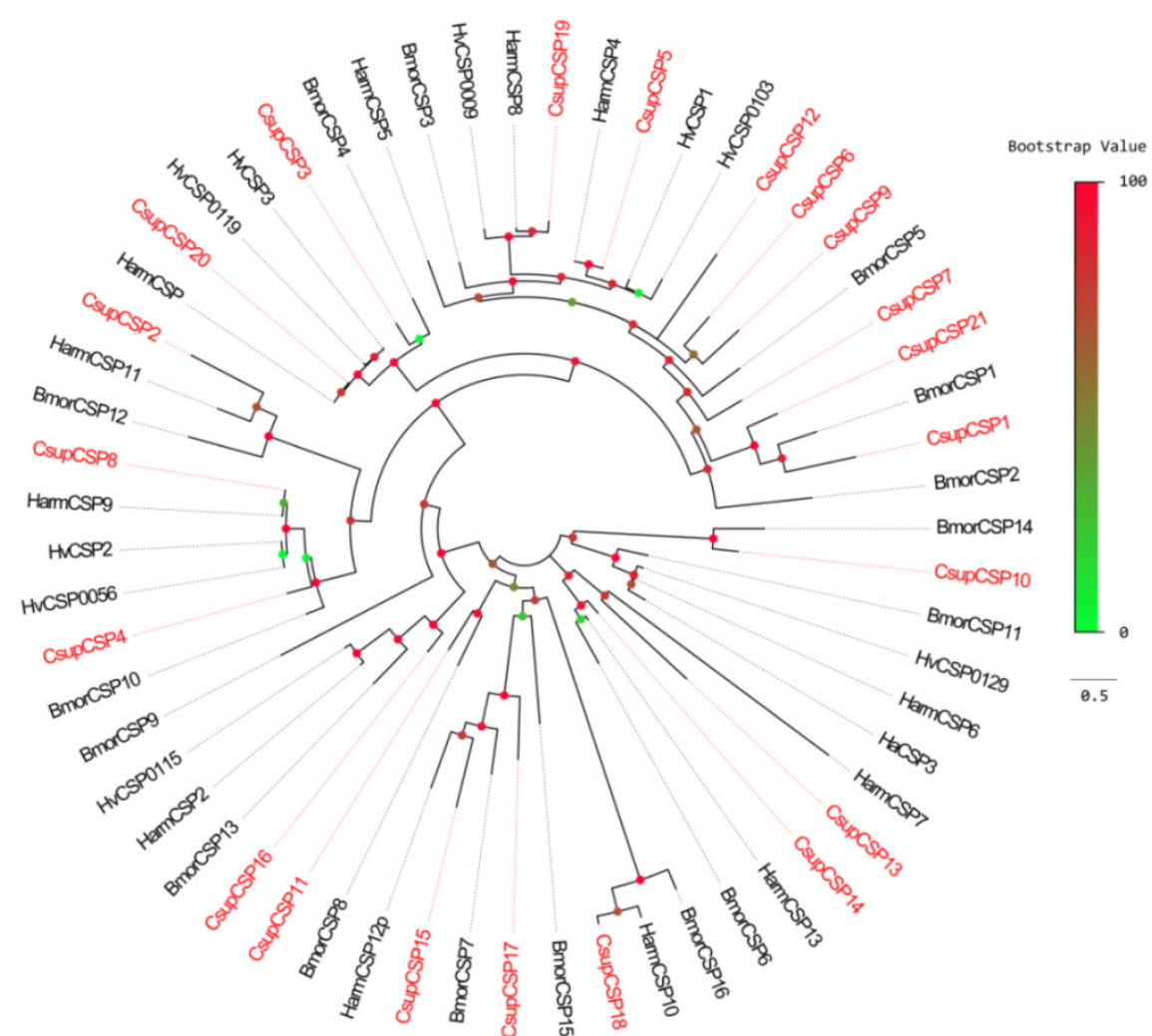

Figure 7. Phylogenetic tree of candidate CsupCSPs with known lepidopteran CSPs. Bmor: B. mori, Hv: H. virescens. 
Table 4 Unigenes of candidate odorant binding proteins

\begin{tabular}{|c|c|c|c|c|c|c|}
\hline Gene name & $\begin{array}{l}\text { Lengt } \\
\mathrm{h}(\mathrm{nt})\end{array}$ & $\begin{array}{l}\text { ORF } \\
(\mathrm{aa})\end{array}$ & $\begin{array}{l}\text { Unigene } \\
\text { reference }\end{array}$ & Status & $\begin{array}{l}\text { Signal } \\
\text { Peptide }\end{array}$ & Evalue BLASTx best hit \\
\hline CsupGOBP1 & 907 & 173 & CL3430.Contig1 & Complete ORF & $\mathrm{Y}$ & 3.0E-97 gb|ACJ07126.1 | general odorant binding protein 1 [Chilo suppressalis] \\
\hline CsupGOBP2 & 1068 & 162 & Unigene17531 & Complete ORF & $\mathrm{Y}$ & 1.0E-86 gb | ACJ07120.1 | general odorant binding protein 2 [Chilo suppressalis] \\
\hline CsupPBP1 & 988 & 184 & Unigene28597 & Complete ORF & $\mathrm{N}$ & 1.0E-91 gb|ADK66921.1 | pheromone binding protein 1 [Chilo suppressalis] \\
\hline CsupPBP2 & 4508 & 165 & CL470.Contig5 & Complete ORF & $\mathrm{Y}$ & 1.0E-89 gb|ACJ07123.1 | pheromone binding protein 2 [Chilo suppressalis] \\
\hline CsupPBP3 & 731 & 170 & Unigene28180 & Complete ORF & $\mathrm{Y}$ & 1.0E-91 gb|ADL09140.1| pheromone binding protein 3 [Chilo suppressalis] \\
\hline CsupPBP4 & 689 & 165 & Unigene31774 & Complete ORF & $\mathrm{Y}$ & 1.0E-38 gb|ADT78499.1 | pheromone binding protein 5 [Ostrinia nubilalis] \\
\hline CsupOBP1 & 1224 & 351 & CL5570.Contig1 & Complete ORF & $\mathrm{Y}$ & 6.0E-21 dbj|BAI82446.1 | odorant binding protein 6 [Delia antiqua] \\
\hline CsupOBP2 & 1212 & 251 & Unigene24952 & Complete ORF & $\mathrm{Y}$ & $\begin{array}{l}\text { 1.0E-14 gb|ADD71058.1 | odorant-binding protein [Chilo suppressalis] } \\
2\end{array}$ \\
\hline CsupOBP3 & 917 & 242 & Unigene28587 & Complete ORF & $\mathrm{Y}$ & 5.0E-09 gb|EFA09155.1 | odorant binding protein 22 [Tribolium castaneum] \\
\hline CsupOBP4 & 1038 & 194 & CL2795.Contig2 & Complete ORF & $\mathrm{Y}$ & 1.0E-44 gb |EHJ77172.1 | odorant binding protein [Danaus plexippus] \\
\hline CsupOBP5 & 753 & 184 & Unigene31885 & Complete ORF & $\mathrm{Y}$ & $\begin{array}{l}\text { 6.0E-35 emb |CAX63249.1| odorant-binding protein SaveOBP4 precursor, partial } \\
\text { [Sitobion avenae] }\end{array}$ \\
\hline CsupOBP6 & 1201 & 184 & Unigene22461 & Complete ORF & $\mathrm{Y}$ & $\begin{array}{l}\text { 1.0E-40 ref |NP_001159621.1 | odorant binding protein LOC100307012 precursor } \\
\text { [Bombyx mori] }\end{array}$ \\
\hline CsupOBP7 & 599 & 156 & Unigene29832 & Complete ORF & $\mathrm{Y}$ & 2.0E-14 gb|AFI57166.1 | odorant-binding protein 17 [Helicoverpa armigera] \\
\hline CsupOBP8 & 608 & 153 & Unigene31806 & Complete ORF & $\mathrm{Y}$ & 2.0E-79 gb|AER27567.1| odorant binding protein [Chilo suppressalis] \\
\hline CsupOBP9 & 697 & 150 & Unigene13466 & Complete ORF & $\mathrm{Y}$ & 7.0E-27 gb|AAR28762.1| odorant-binding protein [Spodoptera frugiperda] \\
\hline CsupOBP10 & 620 & 147 & Unigene17320 & Complete ORF & $\mathrm{Y}$ & 2.0E-37 dbj|BAI44701.1 | odorant binding protein [Bombyx mori] \\
\hline CsupOBP11 & 1046 & 147 & Unigene542 & Complete ORF & $\mathrm{Y}$ & 5.0E-60 gb |AFG72998.1 | odorant-binding protein 1 [Cnaphalocrocis medinalis] \\
\hline CsupOBP12 & 1847 & 145 & Unigene4662 & Complete ORF & $\mathrm{N}$ & 4.0E-45 gb | AFG72998.1 | odorant-binding protein 1 [Cnaphalocrocis medinalis] \\
\hline CsupOBP13 & 1151 & 145 & Unigene15206 & $5^{\prime}$ lost & $\mathrm{Y}$ & 8.0E-60 gb | AFI57166.1 | odorant-binding protein 17 [Helicoverpa armigera] \\
\hline CsupOBP14 & 534 & 140 & Unigene22895 & Complete ORF & $\mathrm{Y}$ & 4.0E-15 gb| ACX53795.1| odorant binding protein [Heliothis virescens] \\
\hline CsupOBP15 & 978 & 139 & Unigene3748 & Complete ORF & $\mathrm{Y}$ & 2.0E-63 gb|AFG73000.1 | odorant-binding protein 2 [Cnaphalocrocis medinalis] \\
\hline CsupOBP16 & 636 & 138 & Unigene2333 & Complete ORF & $\mathrm{Y}$ & 1.0E-38 gb|AFI57167.1 | odorant-binding protein 18 [Helicoverpa armigera] \\
\hline CsupOBP17 & 1503 & 133 & CL2095.Contig1 & Complete ORF & $\mathrm{Y}$ & 2.0E-24 gb|EFA04687.1 | odorant binding protein 08 [Tribolium castaneum] \\
\hline CsupOBP18 & 563 & 129 & CL5651.Contig2 & Complete ORF & $\mathrm{N}$ & 2.0E-29 gb|AFG72998.1 | odorant-binding protein 1 [Cnaphalocrocis medinalis] \\
\hline CsupOBP19 & 2038 & 121 & CL5839.Contig1 & $3^{\prime}$ lost & $\mathrm{Y}$ & 5.0E-44 gb|EHJ65653.1| odorant-binding protein 1 [Danaus plexippus] \\
\hline CsupOBP20 & 387 & 113 & Unigene36476 & $3^{\prime}$ lost & $\mathrm{Y}$ & 2.0E-41 gb |AFD34173.1 | odorant binding protein 5 [Argyresthia conjugella] \\
\hline
\end{tabular}

Table 5 Unigenes of candidate chemosensory proteins

\begin{tabular}{|c|c|c|c|c|c|c|c|}
\hline Gene name & $\begin{array}{l}\text { Length } \\
\text { (nt) }\end{array}$ & $\begin{array}{l}\text { ORF } \\
\text { (aa) }\end{array}$ & $\begin{array}{l}\text { Unigene } \\
\text { reference }\end{array}$ & Status & $\begin{array}{l}\text { Signal } \\
\text { Pep- } \\
\text { tides }\end{array}$ & Evalue & BLASTx best hit \\
\hline CsupCSP1 & 727 & 189 & Unigene35502_All & Complete ORF & $\mathrm{Y}$ & $2.00 \mathrm{E}-37$ & gb|ACX53806.1|chemosensory protein [Heliothis virescens] \\
\hline CsupCSP2 & 3757 & 151 & CL5573.Contig2_All & Complete ORF & Y & $6.00 \mathrm{E}-38$ & gb|EHJ76401.1|chemosensory protein CSP1 [Danaus plexippus] \\
\hline CsupCSP3 & 1279 & 131 & Unigene21279_All & Complete ORF & Y & $1.00 \mathrm{E}-52$ & gb|ACX53804.1|chemosensory protein [Heliothis virescens] \\
\hline CsupCSP4 & 1782 & 129 & Unigene13693_All & Complete ORF & Y & $1.00 \mathrm{E}-17$ & dbj|BAF91712.1|chemosensory protein [Papilio xuthus] \\
\hline CsupCSP5 & 510 & 128 & CL2398.Contig1_All & Complete ORF & Y & $1.00 \mathrm{E}-68$ & gb|AFR92093.1|chemosensory protein 9 [Helicoverpa armigera] \\
\hline CsupCSP6 & 710 & 126 & Unigene2439_All & Complete ORF & Y & $6.00 \mathrm{E}-47$ & dbj|BAF91714.1|chemosensory protein [Papilio xuthus] \\
\hline CsupCSP7 & 625 & 126 & Unigene31984_All & Complete ORF & Y & $3.00 \mathrm{E}-43$ & dbj|BAG71914.1 |chemosensory protein 4a [Papilio xuthus] \\
\hline CsupCSP8 & 503 & 126 & Unigene39861_All & Complete ORF & $\mathrm{Y}$ & $5.00 \mathrm{E}-65$ & gb|AAM77040.1|chemosensory protein 2 [Heliothis virescens] \\
\hline CsupCSP9 & 481 & 124 & Unigene39944_All & Complete ORF & Y & $2.00 \mathrm{E}-64$ & $\mathrm{gb} \mid \mathrm{ACX} 53727.1$ |chemosensory protein [Heliothis virescens] \\
\hline CsupCSP10 & 577 & 123 & CL4176.Contig1_All & Complete ORF & $\mathrm{Y}$ & $1.00 \mathrm{E}-53$ & dbj|BAG71921.1 | chemosensory protein 13 [Papilio xuthus] \\
\hline CsupCSP11 & 726 & 123 & Unigene13345_All & Complete ORF & $\mathrm{Y}$ & $5.00 \mathrm{E}-46$ & dbj|BAF91716.1|chemosensory protein [Papilio xuthus] \\
\hline CsupCSP12 & 456 & 123 & Unigene25770_All & Complete ORF & Y & $9.00 \mathrm{E}-35$ & gb|EHJ78408.1|chemosensory protein [Danaus plexippus] \\
\hline CsupCSP13 & 769 & 121 & CL1604.Contig1_All & Complete ORF & Y & $5.00 \mathrm{E}-21$ & gb|EHJ73331.1|chemosensory protein 12 [Danaus plexippus] \\
\hline CsupCSP14 & 1272 & 121 & CL5801.Contig1_All & Complete ORF & Y & $3.00 \mathrm{E}-56$ & gb|ACX53719.1|chemosensory protein [Heliothis virescens] \\
\hline CsupCSP15 & 503 & 121 & Unigene24547_All & Complete ORF & $\mathrm{Y}$ & $9.00 \mathrm{E}-29$ & $\begin{array}{l}\text { ref|NP_001037068.1 | chemosensory protein } 7 \text { precursor [Bombyx } \\
\text { mori] }\end{array}$ \\
\hline CsupCSP16 & 536 & 121 & Unigene28390_All & Complete ORF & $\mathrm{Y}$ & $3.00 \mathrm{E}-41$ & dbj|BAF91717.1|chemosensory protein [Papilio xuthus] \\
\hline CsupCSP17 & 2686 & 120 & CL4999.Contig1_All & Complete ORF & Y & 3.00E-27 & dbj|BAG71919.1|chemosensory protein 11b [Papilio xuthus] \\
\hline CsupCSP18 & 1153 & 109 & Unigene15508_All & Complete ORF & $\mathrm{Y}$ & $1.00 \mathrm{E}-48$ & dbj|BAF91720.1 | chemosensory protein [Papilio xuthus] \\
\hline CsupCSP19 & 367 & 97 & CL2398.Contig2_All & $5^{\prime}$ lost & $\mathrm{N}$ & $1.00 \mathrm{E}-51$ & $\mathrm{gb}$ |AFR92095.1|chemosensory protein 11 [Helicoverpa armigera] \\
\hline CsupCSP20 & 306 & 95 & Unigene38877_All & $5^{\prime}$ lost & $\mathrm{N}$ & $1.00 \mathrm{E}-50$ & gb|AAK53762.1|chemosensory protein [Helicoverpa armigera] \\
\hline CsupCSP21 & 268 & 89 & Unigene39576_All & $5^{\prime}, 3^{\prime}$ lost & $\mathrm{N}$ & $1.00 \mathrm{E}-39$ & $\mathrm{gb}|\mathrm{ACX} 53806.1|$ chemosensory protein [Heliothis virescens] \\
\hline
\end{tabular}


Tissue- and Sex- specific Expression of Candidate $C$. suppressalis $O R$ and IR genes

The expression patterns of the candidate 7 ORs and 20 IRs in male antennae, female antennae and legs were analyzed by semi-quantitative reverse transcription PCR. Results for all of these genes are listed in Figure 8.

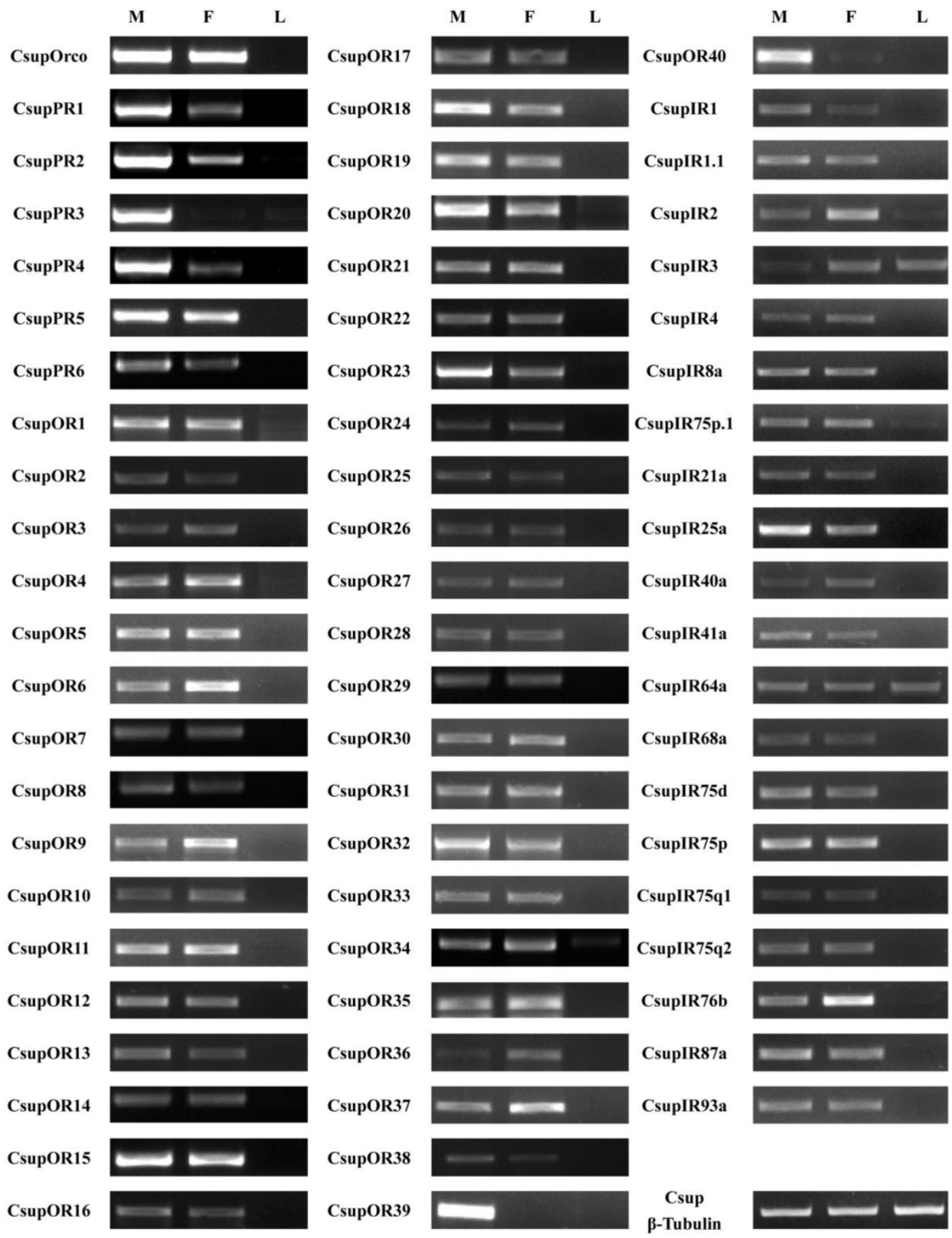

Figure 8. Tissue- and sex- specific expressions of candidates CsupORs and CsupIRs. M: male antennae, F: female antennae, L: legs. 
All 47 ORs were successfully detected as expressed in our semi-quantitative RT-PCR analysis. These results indicated that these candidate ORs are expressed in the olfactory organ antennae, but not in non-olfactory organs such as legs. Of the six candidate PRs, only CsupPR3 was found to be exclusive to the male antennae. CsupPR1, 2 and 4 have expression detected in both male and female antennae, but the amounts in male antennae were significantly higher than in female antennae. Of the candidate ORs, two sequences (CsupOR39 and CsupOR40) showed a male-specific expression. The remaining ORs were expressed in both sexes, with some differential expression in male or female antennae. Compared with ORs, the candidate IRs showed no big differences between male and female. Additionally, CsupIR3 and CsupIR64a have considerable expression in leg, at similar levels, based upon luminance of the bands in the agarose gels. CsupIR2 and CsupIR75p.1 are also weakly expressed in legs.

\section{Discussion}

Our antennal transcriptome sequencing provides a dataset of 47 ORs, 20 IRs, 26 OBPs and 21 CSPs. All of the previously annotated C. suppressalis chemosensory genes available in NCBI were identified in our dataset. Compared to the antennal transcriptomes of M.sexta with 47 ORs [27], C. pomonella with 43 ORs [28] and Helicoverpa armigera with 47 ORs [4], our OR dataset of 47 sequences is of similar quantity. The neuroanatomical study of C. suppressalis' close relative, the European corn borer (ECB) (Ostrinia nubilalis) suggested that there are 64 glomeruli in the antenna lobe of male and female ECB [29]. If the logic holds true that one olfactory receptor type is expressed in OSN type and axonal projects of different OSNs expressing the same olfactory receptor converge on the same antennal lobe glomeruli, our OR dataset of 47 sequences is quite reasonable, for some glomeruli should also be innervated by OSNs expressing other classes of chemoreceptors such as ionotropic receptors [18].

We identified 6 candidate PR genes by their similarities to PRs in other Lepidopterans and physiologic analysis. But the expression profiles of these six sequences showed that not all of them are exclusive in male antenna. Although PR expression in Bombyx mori and some Lepidoptera have been shown to be restricted to male antennae [30,31], some recent studies gave examples of PR genes expressed in both sexes. In the antennal transcriptome of Helicoverpa armigera, 6 candidate PR genes were discovered; two of them, HarmOR6 and HarmOR13, display expression in male and female antenna [4]. Two candidate PRs identified in S. littoralis were also found to be expressed in an- tennae of both sexes [32]. Obviously, this phenomenon cannot be simply ascribed to differences between species. A physiology and morphology study suggested that $S$. littoralis females detect their own pheromone. The rationale behind female pheromone perception has been proposed to be optimization of pheromone production and spatial dispersion of females over host plants [33, 34].

Ionotropic receptors represent a new member of the chemosensory receptor family, and were first discovered in D. melanogaster [18] through genome analyses. The Ionotropic Receptor family is a variant iGluR subfamily. Animal iGluRs have been best characterized for their essential roles in synaptic transmission as receptors for the excitatory neurotransmitter glutamate [35, 36]. IRs share a considerable degree of commonality with the typical iGluRs: first, they are all located to specialized distal membrane domains of neuronal dendrites (OSN cilia and post-synaptic membranes, respectively); secondly, they responds to binding of extracellular ligands (volatile odorants and neurotransmitter); thirdly, they are comprised of multimeric functional complexes (IR8a/25a co-express with other cell-type specific IRs and iGluRs are formed of heteromeric subunits) [20]. It is easy to conjecture that the IR arose from an iGluR with a change in expression localization from an interneuron to a sensilla neuron [20]. In our study, we found 20 IR candidates in C. suppressalis antennae including two co-receptors, IR8a and IR25a. Compared to ORs, the IR family is relatively conserved both in sequence and expression pattern. Among the 20 CsupIRs we discovered, 15 sequences have orthologs found in Dmel/Bmor/Slit IRs; the expression levels have no significant difference between male and female antenna, which is similar to results in $S$. littoralis IRs [37] and H. armigera IRs [4]. Considering the relatively high sequence conservation and similarities in expression, the functions of CsupIRs are probably conserved as IRs in other Lepidoptera.

\section{Conclusion}

Our goal for this study was to identify genes potentially involved in olfactory signal detection in $C$. suppressalis, and this is well met by a repertoire of 47 ORs, 20 IRs, 26 OBPs and 21 CSPs. Our approach has been proved to be successful in identifying low-expressing chemosensory receptor genes, especially in a non-model pest species without an available genome sequence. Our findings make it possible for future research of the olfactory system of C. suppressalis at the molecular level, and provide information for comparative and functional genomic analyses of related species. 


\section{Materials and Methods}

\section{Insects}

C. suppressalis were obtained from a laboratory colony maintained at the Institute of Plant Protection, Chinese Academy of Agricultural Sciences, Beijing, China. Larvae were reared on an artificial diet at $28 \pm 1^{\circ} \mathrm{C}, 70 \pm 5 \%$ relative humidity, and under a photoperiod of 14:10 (light: dark). After pupation, male and female pupae were separated for adult eclosion in cages kept at $30 \pm 1^{\circ} \mathrm{C}, 80 \pm 5 \% \mathrm{RH}$ and 16: 8 light/dark cycles, and were fed with $10 \%$ sugar solution. Antennae of unmated male or female individuals were collected 1-3 days after eclosion and immediately frozen in liquid nitrogen, and stored at $-70^{\circ} \mathrm{C}$ until extraction. Antennae were pulled off with tweezers grasped at the very root of the antennae, in order to reserve the intact structure of antennae.

\section{RNA preparing}

Frozen antennae were crushed in a liquid nitrogen cooled vitreous homogenizer and total RNA was extracted using TRIzol reagent (Invitrogen, Carlsbad, CA, USA) following the manufacturer's instructions. Residual DNA in total RNA was removed by DNase I (Promega, Madison, WI, USA). Total RNA was dissolved in RNase-free water and RNA integrity was verified by gel electrophoresis. RNA quantity was determined on a Nanodrop ND-2000 spectrophotometer (NanoDrop products, Wilmington, DE, USA).

\section{cDNA library construction and sequencing}

Ten micrograms of total RNA extracted from approximately 500 antennae of 1-3 day old adult male or female moths was used to isolate poly-A RNA using oligo(dT) magnetic beads. Poly-A RNA of each sample was digested into short fragments by fragmentation buffer. Random hexamers were used for first-strand cDNA, followed by second-strand cDNA synthesis using RNase $\mathrm{H}$ and DNA polymerase I. These dual-strand DNA samples were treated with T4 DNA Polymerase and T4 Polynucleotide Kinase for end-repairing and dA-tailing, followed by adaptor ligation to the dsDNA's dA tail using T4 DNA ligase. Then bands of insert length around 200bp was collected by $2 \%$ agarose gel electrophoresis and purified with QIAquick Gel Extraction Kit (Qiagen, Hilden, Germany), and used as templates for PCR amplification to create the cDNA library.

The library was pair-end sequenced using PE90 strategy (paired-end reads of 90 base pairs per read) on Illumina HiSeq ${ }^{\mathrm{TM}} 2000$ (Illumina, San Diego, CA, USA) at the Beijing Genome Institute (Shenzhen, China). Different libraries were sequenced in one lane and raw-reads were sorted by barcodes in the se- quencing adaptor.

\section{Assembly}

The raw-reads were treated to generate clean-read datasets by the following procedure. First, reads with adaptors or containing unknown nucleotides (Ns) more than 5\% were removed directly. Secondly, low-quality reads containing more than $20 \%$ suspect-nucleotides of Phred Quality Score less than 10 were filtered out. Finally, both ends of reads were evaluated to trim unreliable ends containing more than 3 successive suspect-nucleotides. Each clean-read dataset of male and female antenna was feed to Trinity r2012-06-08 [38] separately for De novo assembly using paired reads mode and default parameters. Then the Trinity outputs were clustered by TGICL [39]. The consensus cluster sequences and singletons make up the unigenes dataset.

\section{Functional annotation}

The annotation of unigenes were performed by NCBI blastx against a pooled database of non-redundant (nr) and SwissProt protein sequences with e-value $<1$ e-5. The blast results were then imported into Blast2GO [40] pipeline for GO Annotation. Protein coding region prediction was performed by OrfPredictor [41] according to the blast result. The signal peptide of the protein sequences were predicted using SignalP 4.0 [41] server version (http://www.cbs.dtu.dk/services/SignalP/) with default parameters. The transmembrane-domains of annotated genes were predicted using TMHMM [43] server version2.0 (http://www.cbs.dtu.dk/ services/TMHMM) with the new model.

\section{Phylogenetic analyses}

The phylogenetic reconstruction of C. suppressalis chemosensory genes was performed according to our previous research [4]. Amino acid sequences were aligned using Clustal Omega [44]. Phylogenetic trees were constructed by the neighbor-joining method, with Jones-Taylor-Thornton (JTT) amino acid substitution model, as implemented in MEGA5.2 software. Node support was assessed using a bootstrap procedure of 1000 replicates.

\section{Expression analysis by semi-quantitative reverse transcription PCR}

Semi-quantitative reverse transcription PCR was performed to verify the expression of candidate chemosensory genes. Tissue samples were collected from $C$. suppressalis adult 1 day after eclosion for 3 biological replicates and total RNA were extracted as mentioned above. The cDNA was synthesized from total RNA using RevertAid First Strand cDNA Synthesis Kit (Thermo Scientific, Waltham, MA, USA) 
with the gDNA removal procedure performed. Gene-specific primers were designed by Primer3 tool (http://frodo.wi.mit.edu/primer3/) and sequences are available in Additional File 2: supplementary material S2. Taq MasterMix (CWBIO, Beijing, China) was used for PCR reactions under general 3-step amplification of $94{ }^{\circ} \mathrm{C}$ for $30 \mathrm{~s}, 55-60{ }^{\circ} \mathrm{C}$ for $30 \mathrm{~s}, 72{ }^{\circ} \mathrm{C}$ for 30s. The PCR cycle-numbers were adjusted respectively for each gene. For most chemosensory genes, cycle-numbers were range from 30 to 34, but for some high-express-level genes like actin and Orco, cycle-numbers were reduced to 25 to 29 . PCR products were run on a $2 \%$ agarose gel and verified by DNA sequencing.

\section{Abbreviations}

iGluR: ionotropic glutamate receptor; OR: odorant receptor; IR: ionotropic receptor; PR: pheromone receptor; PBP: pheromone binding protein; GOBP: general odorant binding protein; OBP: odorant binding protein; CSP: chemosensory protein; SNMP: sensory neuron membrane protein; GO: gene ontology; FPKM : fragments per kb per million fragments; FDR: false discovery rate; JTT: Jones-Taylor-Thornton amino acid substitution model.

\section{Supplementary Material}

Additional File 1:

Supplementary Material S1 Amino acid sequences of C. suppressalis olfactory genes.

http://www.ijbs.com/v10p0846s1.fa

Additional File 2:

Supplementary Material S2 Primers used in the semi-quantitative RT-PCR analysis.

http://www.ijbs.com/v10p0846s2.xlsx

\section{Acknowledgements}

We thank Dr. Yunhe Li (Institute of Plant Protection, Chinese Academy of Agricultural Sciences) for providing the insects. This work was supported by National Basic Research Program of China (973 Program, 2012CB114104), National Natural Science Foundation of China (31230062) and International Science \& Technology Cooperation Program of China (2013DFG32230).

\section{Data Deposition}

The clean reads of the C. suppressalis antennal transcriptome were stored in the NCBI SRA database, under the accession number of SRX497236 and SRX497239.

\section{Competing Interests}

The authors have declared that no competing interest exists.

\section{References}

1. Sato K, Touhara K. Insect olfaction: receptors, signal transduction, and behavior. Results and problems in cell differentiation. 2009; 47: 121-38. doi:10.1007/400_2008_10.

2. Gao Q, Chess A. Identification of candidate Drosophila olfactory receptors from genomic DNA sequence. Genomics. 1999; 60: 31-9. doi:10.1006/geno.1999.5894.

3. Zhao XC, Tang QB, Berg BG, Liu Y, Wang YR, Yan FM, et al. Fine structure and primary sensory projections of sensilla located in the labial-palp pit organ of Helicoverpa armigera (Insecta). Cell and tissue research. 2013; 353: 399-408. doi:10.1007/s00441-013-1657-z.

4. Liu Y, Gu S, Zhang Y, Guo Y, Wang G. Candidate olfaction genes identified within the Helicoverpa armigera Antennal Transcriptome. PloS one. 2012; 7: e48260. doi:10.1371/journal.pone.0048260.

5. Klein U. Sensillum-lymph proteins from antennal olfactory hairs of the moth Antheraea polyphemus (Saturniidae). Insect biochemistry. 1987; 17: 1193-204. doi: 10.1016/0020-1790(87)90093-X

6. Krieger J, Breer H. Olfactory reception in invertebrates. Science. 1999; 286: 720-3. doi: 10.1126/science. 286.5440.720

7. Campanacci V, Krieger J, Bette S, Sturgis JN, Lartigue A, Cambillau C, et al. Revisiting the specificity of Mamestra brassicae and Antheraea polyphemus pheromone-binding proteins with a fluorescence binding assay. The Journal of biological chemistry. 2001; 276: 20078-84. doi:10.1074/jbc.M100713200.

8. Pelosi P, Maida R. Odorant-binding proteins in insects. Comparative biochemistry and physiology Part B, Biochemistry \& molecular biology. 1995; 111: 503-14. doi: 10.1016/0305-0491(95)00019-5

9. Pelosi P, Zhou JJ, Ban LP, Calvello M. Soluble proteins in insect chemical communication. Cellular and molecular life sciences : CMLS. 2006; 63: 1658-76. doi:10.1007/s00018-005-5607-0.

10. Laughlin JD, Ha TS, Jones DN, Smith DP. Activation of pheromone-sensitive neurons is mediated by conformational activation of pheromone-binding protein. Cell. 2008; 133: 1255-65. doi:10.1016/j.cell.2008.04.046.

11. Kim MS, Repp A, Smith DP. LUSH odorant-binding protein mediates chemosensory responses to alcohols in Drosophila melanogaster. Genetics. 1998; 150: 711-21.

12. Briand L, Swasdipan N, Nespoulous C, Bezirard V, Blon F, Huet JC, et al. Characterization of a chemosensory protein $(A S P 3 c)$ from honeybee (Apis mellifera $L$.) as a brood pheromone carrier. European journal of biochemistry / FEBS. 2002; 269: 4586-96. doi:10.1046/j.1432-1033.2002.03156.x

13. Clyne PJ, Warr CG, Freeman MR, Lessing D, Kim J, Carlson JR. A novel family of divergent seven-transmembrane proteins: candidate odorant receptors in Drosophila. Neuron. 1999; 22: 327-38. doi:10.1016/S0896-6273(00)81093-4

14. Benton R, Sachse S, Michnick SW, Vosshall LB. Atypical membrane topology and heteromeric function of Drosophila odorant receptors in vivo. PLoS biology. 2006; 4: e20. doi:10.1371/journal.pbio.0040020.

15. Sato $K$, Pellegrino $M$, Nakagawa $T$, Nakagawa T, Vosshall LB, Touhara $K$. Insect olfactory receptors are heteromeric ligand-gated ion channels. Nature. 2008; 452: 1002-6. doi:10.1038/nature06850.

16. Wicher D, Schafer R, Bauernfeind R, Stensmyr MC, Heller R, Heinemann SH, et al. Drosophila odorant receptors are both ligand-gated and cyclic-nucleotide-activated cation channels. Nature. 2008; 452: 1007-11. doi:10.1038/nature06861.

17. Larsson MC, Domingos AI, Jones WD, Chiappe ME, Amrein H, Vosshall LB. Or $83 b$ encodes a broadly expressed odorant receptor essential for Drosophila olfaction. Neuron. 2004; 43: 703-14. doi:10.1016/j.neuron.2004.08.019.

18. Benton R, Vannice KS, Gomez-Diaz C, Vosshall LB. Variant ionotropic glutamate receptors as chemosensory receptors in Drosophila. Cell. 2009; 136: 149-62. doi:10.1016/j.cell.2008.12.001.

19. Abuin L, Bargeton B, Ulbrich MH, Isacoff EY, Kellenberger S, Benton R. Functional architecture of olfactory ionotropic glutamate receptors. Neuron. 2011; 69: 44-60. doi:10.1016/j.neuron.2010.11.042.

20. Croset V, Rytz R, Cummins SF, Budd A, Brawand D, Kaessmann H, et al. Ancient protostome origin of chemosensory ionotropic glutamate receptors and the evolution of insect taste and olfaction. PLoS genetics. 2010; 6: e1001064. doi:10.1371/journal.pgen.1001064.

21. Chiu J, DeSalle R, Lam HM, Meisel L, Coruzzi G. Molecular evolution of glutamate receptors: a primitive signaling mechanism that existed before plants and animals diverged. Molecular biology and evolution. 1999; 16: 826-38.

22. Zhou JJ, He XL, Pickett JA, Field LM. Identification of odorant-binding proteins of the yellow fever mosquito Aedes aegypti: genome annotation and comparative analyses. Insect molecular biology. 2008; 17: 147-63. doi:10.1111/j.1365-2583.2007.00789.x.

23. Hekmat-Scafe DS, Scafe CR, McKinney AJ, Tanouye MA. Genome-wide analysis of the odorant-binding protein gene family in Drosophila melanogaster. Genome research. 2002; 12: 1357-69. doi:10.1101/gr.239402.

24. Zhou JJ, Kan Y, Antoniw J, Pickett JA, Field LM. Genome and EST analyses and expression of a gene family with putative functions in insect

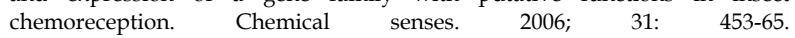
doi:10.1093/chemse/bji050.

25. Rogers ME, Krieger J, Vogt RG. Antennal SNMPs (sensory neuron membrane proteins) of Lepidoptera define a unique family of invertebrate CD36-like proteins. Journal of neurobiology. 2001; 49: 47-61. doi:10.1002/neu.1065 
26. Benton R, Vannice KS, Vosshall LB. An essential role for a CD36-related receptor in pheromone detection in Drosophila. Nature. 2007; 450: 289-93. doi:10.1038/nature06328

27. Grosse-Wilde E, Kuebler LS, Bucks S, Vogel H, Wicher D, Hansson BS, Antennal transcriptome of Manduca sexta. Proceedings of the National Academy of Sciences of the United States of America. 2011; 108: 7449-54. doi:10.1073/pnas.1017963108

28. Bengtsson JM, Trona F, Montagne N, Anfora G, Ignell R, Witzgall P, et al. Putative chemosensory receptors of the codling moth, Cydia pomonella, identified by antennal transcriptome analysis. PloS one. 2012; 7: e31620. doi:10.1371/journal.pone.0031620.

29. Karpati Z, Dekker T, Hansson BS. Reversed functional topology in the antennal lobe of the male European corn borer. The Journal of experimental biology. 2008; 211: 2841-8. doi:10.1242/jeb.017319.

30. Mitsuno H, Sakurai T, Murai M, Yasuda T, Kugimiya S, Ozawa R, et al. Identification of receptors of main sex-pheromone components of three Lepidopteran species. The European journal of neuroscience. 2008; 28: 893-902. doi:10.1111/j.1460-9568.2008.06429.x.

31. Sakurai T, Nakagawa T, Mitsuno H, Mori H, Endo Y, Tanoue S, et al. Identification and functional characterization of a sex pheromone receptor in the silkmoth Bombyx mori. Proceedings of the National Academy of Sciences of the United States of America. 2004; 101: 16653-8. doi:10.1073/pnas.0407596101.

32. Legeai F, Malpel S, Montagné N, Monsempes C, Cousserans F, Merlin C, et al. An Expressed Sequence Tag collection from the male antennae of the Noctuid moth Spodoptera littoralis: a resource for olfactory and pheromone detection research. BMC Genomics. 2011; 12: 86. doi:10.1186/1471-2164-12-86.

33. Palanaswamy $\mathrm{P}$, Seabrook W. Behavioral responses of the female eastern spruce budworm Choristoneura fumiferana (Lepidoptera, Tortricidae) to the sex pheromone of her own species. Journal of Chemical Ecology. 1978; 4: 649-55.

34. Schneider D, Schulz S, Priesner E, Ziesmann J, Francke W. Autodetection and chemistry of female and male pheromone in both sexes of the tiger moth Panaxia quadripunctaria. Journal of Comparative Physiology A. 1998; 182: 153-61. doi:10.1007/s003590050166.

35. Gereau IV RW, Swanson GT. The glutamate receptors. Springer. 2008.

36. Madden DR. The structure and function of glutamate receptor ion channels. Nature reviews Neuroscience. 2002; 3: 91-101. doi:10.1038/nrn725.

37. Olivier V, Monsempes C, Francois MC, Poivet E, Jacquin-Joly E. Candidate chemosensory ionotropic receptors in a Lepidoptera. Insect molecular biology. 2011; 20: 189-99. doi:10.1111/j.1365-2583.2010.01057.x.

38. Grabherr MG, Haas BJ, Yassour M, Levin JZ, Thompson DA, Amit I, et al. Full-length transcriptome assembly from RNA-Seq data without a reference genome. Nature biotechnology. 2011; 29: 644-52. doi:10.1038/nbt.1883.

39. Pertea G, Huang X, Liang F, Antonescu V, Sultana R, Karamycheva S, et al. TIGR Gene Indices clustering tools (TGICL): a software system for fast clustering of large EST datasets. Bioinformatics. 2003; 19: 651-2. doi:10.1093/bioinformatics/btg034

40. Conesa A, Götz S, García-Gómez JM, Terol J, Talón M, Robles M. Blast2GO: a universal tool for annotation, visualization and analysis in functional genomics research. Bioinformatics. 2005; 21: 3674-6. doi: 10.1093/bioinformatics/bti610

41. Min XJ, Butler G, Storms R, Tsang A. OrfPredictor: predicting protein-coding regions in EST-derived sequences. Nucleic acids research. 2005; 33: W677-80. doi:10.1093/nar/gki394.

42. Petersen TN, Brunak S, von Heijne G, Nielsen H. SignalP 4.0: discriminating signal peptides from transmembrane regions. Nature methods. 2011; 8: 785-6. doi:10.1038/nmeth.1701.

43. Krogh A, Larsson B, von Heijne G, Sonnhammer EL. Predicting transmembrane protein topology with a hidden Markov model: application to complete genomes. Journal of molecular biology. 2001; 305: 567-80. doi:10.1006/jmbi.2000.4315.

44. Sievers F, Wilm A, Dineen D, Gibson TJ, Karplus K, Li W, et al. Fast, scalable generation of high-quality protein multiple sequence alignments using Clustal Omega. Molecular systems biology. 2011; 7: 539. doi:10.1038/msb.2011.75. 